Check for updates

Cite this: Mater. Adv., 2021, 2, 2132

Received 26th December 2020, Accepted 4th February 2021

DOI: 10.1039/d0ma01017k

rsc.li/materials-advances

\title{
Achieving ultra-narrow bandgap non-halogenated non-fullerene acceptors via vinylene $\pi$-bridges for efficient organic solar cells $\dagger$
}

\author{
Jiefeng Hai, $\ddagger^{\text {ab }}$ Siwei Luo, $\ddagger^{c}$ Han Yu, $\ddagger^{c}$ Honggang Chen, ${ }^{d}$ Zhenhuan Lu, (D) ${ }^{b}$ \\ Ling Li, ${ }^{b}$ Yingping Zou (D) *d and He Yan (D)*ac
}

\begin{abstract}
The field of organic solar cells (OSCs) has witnessed rapid progress in the last two years, due to the emergence of high-performance $Y$-series non-fullerene acceptors (NFAs). To further extend this family of the $Y$-series NFAs and tune their optoelectronic properties, herein, we developed a family of the $\mathrm{Y}$-series NFAs (namely BTP-1V, BTP-2V, BTP-3V, and BTP-4V) by inserting vinylene $\pi$-bridges between the $Y$-series central fused core and non-halogenated end group IC. The incorporation of vinylene $\pi$-bridges can effectively reduce the bandgap of these NFAs with a significantly red-shifted absorption edge approaching $1020 \mathrm{~nm}$. The optimized OSC device based on PBDB-T/BTP-1V demonstrated an excellent power conversion efficiency (PCE) of $11.03 \%$ with an open-circuit voltage $\left(V_{\text {oc }}\right)$ of $0.84 \mathrm{~V}$, a short-circuit current density of $20.86 \mathrm{~mA} \mathrm{~cm}^{-2}$, a fill factor of 0.63 , and an energy loss of $0.58 \mathrm{eV}$. It is worth noting that the PCE of over $11 \%$ is the highest value for binary single-junction OSCs based on the non-halogenated NFA with a bandgap below $1.28 \mathrm{eV}$. These results demonstrate that the vinylene $\pi$-bridges strategy strongly regulates the optoelectronic characteristics of NFAs, offering insights into the development of efficient OSCs based on ultra-narrow bandgap Y-series NFAs.
\end{abstract}

\section{Introduction}

Thanks to their light weight, low-cost solution processing, flexibility, semi-transparent outlook and compatibility with high throughput roll-to-roll printing methods, organic solar cells (OSCs) have received extensive research attention. ${ }^{1,2}$ With superior photoinduced electron transfer and suitable phaseseparation property, fullerene derivatives have been widely used as electron acceptors in OSCs for many years. ${ }^{3}$ Due to the intrinsic disadvantages of fullerene acceptors (such as weak photon harvesting ability in the long wavelength range and the limited regulation on their molecular energy levels), the improvement of fullerene-based OSCs faces great challenges. ${ }^{4}$

\footnotetext{
${ }^{a}$ HKUST-Shenzhen Research Institute No. 9 Yuexing 1st Road, Hi-Tech Park, Nanshan Shenzhen 518057, China. E-mail: hyan@ust.hk

${ }^{b}$ Guangxi Key Laboratory of Electrochemical and Magneto-Chemical Functional Materials, Guilin University of Technology, Guilin 541004, Guangxi, China. E-mail:6614034@glut.edu.cn

${ }^{c}$ Department of Chemistry and Hong Kong Branch of Chinese National Engineering Research Center for Tissue Restoration \& Reconstruction, Hong Kong University of Science and Technology (HKUST), Clear Water Bay, Kowloon, Hong Kong, China

${ }^{d}$ College of Chemistry and Chemical Engineering, Central South University,

Changsha 410083, Hunan, China. E-mail: yingpingzou@csu.edu.cn

$\dagger$ Electronic supplementary information (ESI) available. See DOI: 10.1039/ d0ma01017k

\$ These authors contributed equally to this work.
}

Compared with fullerene acceptors, non-fullerene electron acceptors (NFAs) can realize faster carrier separation with negligible driving force, hence achieving lower energy loss $\left(E_{\text {loss }}\right)$ and greatly improved efficiencies. ${ }^{5}$ With the tremendous opportunities to tune their chemical structures, NFAs with acceptor-donor-acceptor (A-D-A) configuration provide a wealth of materials choice for potentially achieving efficient OSCs. ${ }^{6,7}$ Recently, researchers designed acceptor-donor-acceptor'-donor-acceptor (A-DA'D-A) structure NFAs, namely Y6 reaching marvellous efficiencies of $15.7 \% .^{8} \mathrm{Y} 6$ is a sensational NFA material for pioneering in breaking the power conversion efficiency (PCE) bottleneck of $15 \%$ for binary OSCs. The impressive efficiencies in Y-seriesbased OSCs have been attributed to the wide and high photoresponsivity with a relatively low $E_{\text {loss. }}$ Therefore, plenty of works related to this innovative Y-series family have been carried out together with extraordinary PCE surpassing $17 \%$ for binary singlejunction OSCs, showing great potential in commercial application. ${ }^{9-13}$ However, the modification of the Y-series NFAs is primarily limited to side chain and terminal group engineering, which limits the improvement of their absorption region and PCE. ${ }^{14-18}$ Therefore, it is necessary to develop ultra-narrow bandgap (ultra-NBG) NFAs with near-infrared region (NIR) absorption for light harvesting. ${ }^{19}$

Recent studies have suggested that the introduction of $\pi$-bridges between central fused core and terminal electron-withdrawing 
units is an effective strategy to broaden the spectrum for NFA-based OSCs. The extensively used $\pi$-bridge units include alkoxyl or alkylthio thiophene, ${ }^{20,21}$ selenophene, ${ }^{22}$ thiazole, $^{23}$ benzene, ${ }^{24}$ thieno[3,2-b]-thiophene, ${ }^{22}$ and cyclopentadithiophene. ${ }^{25}$ These conjugated units with variant substituent groups usually weaken the planarity of the whole conjugated molecule to some extent. ${ }^{26}$ The carbon-carbon double bond group (vinylene) is used to construct high efficiency D-A narrow bandgap polymers, ${ }^{27-30}$ but was rarely applied in A-DA'D-A type NFA based PSCs. ${ }^{31-33}$ So far, there has been no report on the stepwise introduction of multi vinylene units into the backbone of NFAs to regulate their optoelectronic properties.

With these concerns, we envisioned a general modification strategy for Y6, aiming to broaden absorption nature of Y6 while preserving its other excellent structural features. Therefore, we reported a general synthetic approach toward a series of the Y-series NFAs, featuring ultra-NBG (the optical bandgap $\leq 1.28 \mathrm{eV}$ ) and various electron accepting abilities. The four Y-series NFAs namely BTP-1V, BTP-2V, BTP-3V, and BTP-4V were designed by introducing different numbers of vinylene units between the central electron-deficient fused benzothiadiazole core and the terminal electron-deficient 2-(3-oxo-2, 3-dihydro- $1 H$-inden-1-ylidene)malononitrile (IC) end-capped unit (Fig. 1). When the number of vinylene was one or three, two asymmetrical Y-series NFAs BTP-1V and BTP-3V were obtained. Compared with $\mathrm{Y}^{34}$ and $\mathrm{Y}^{3}{ }^{8}$, asymmetric vinylene modification of the Y-series NFAs simultaneously extends the conjugation length of molecules to enhance the electrondonating properties, generates a large natural dipole moment, and alters the molecular stacking/packing form in the solid state to some extent. ${ }^{35,36}$ The 2-butyloctyl side chain on the nitrogen atom of the centre fused deficient core provides good solubility and crystallinity. These four Y-series NFAs bear the

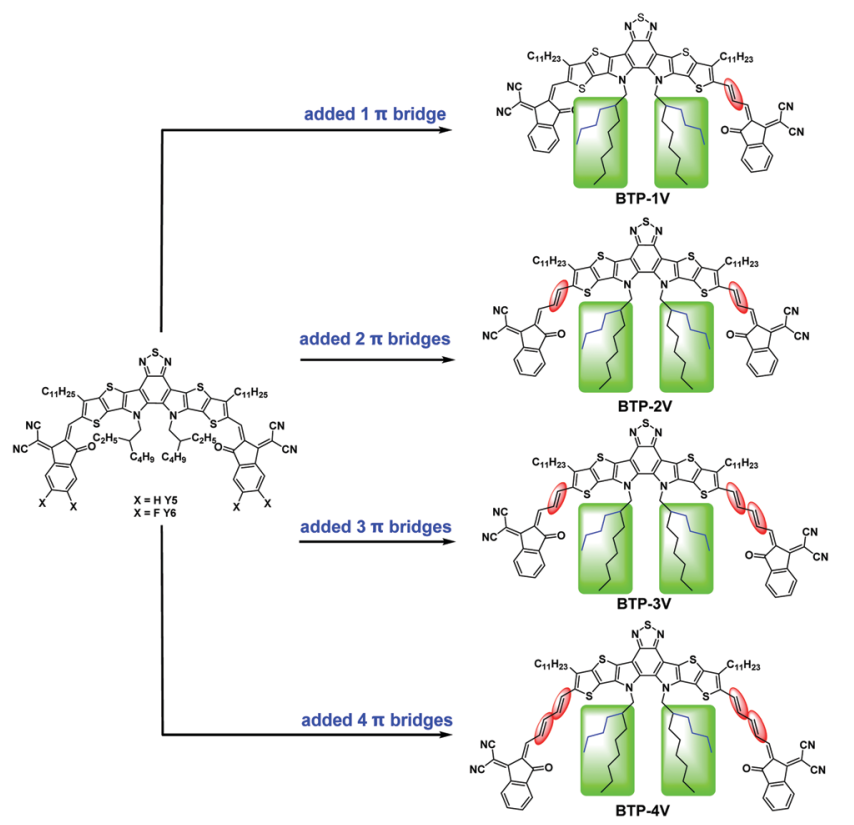

Fig. 1 The molecular structures of BTP-1V, BTP-2V, BTP-3V and BTP-4V. broader absorption range (550-1000 $\mathrm{nm}$ ), the narrower optical bandgap ( $\left.E_{\mathrm{g}}^{\mathrm{opt}}\right)(1.21-1.28 \mathrm{eV}$ ), and the higher occupied molecular orbital (HOMO) energy levels $(-5.54--5.34 \mathrm{eV})$, compared with that of Y6 counterpart. The OSC devices based on these Y-series NFAs blended with the donor polymer PBDB-T or PCE10 were systematically studied. As a result, the best device based on PBDB-T/BTP-1V showed a $V_{\text {oc }}$ of $0.84 \mathrm{~V}$, a $J_{\mathrm{sc}}$ of $20.86 \mathrm{~mA} \mathrm{~cm}^{-2}$, an FF of 0.63 and a PCE of $11.03 \%$, whereas devices based on PCE10/BTP-2V, PCE10/BTP-3V and PCE10/ BTP-4V achieved PCEs of $7.87 \%, 2.04 \%$, and $1.04 \%$, respectively. BTP-1V-based devices exhibited the best value of PCE in these ultra-NBG NFAs-based devices. The lower HOMO energy level of BTP-1V, the more balanced charge transport and the better phase separation morphology in the PBDB-T/BTP-1V blend were found to be major reasons for the superior performance of BTP-1V-based devices, compared with those of the other BTP-V-based devices. To the best of our knowledge, the PCE of over $11 \%$ is the highest value of binary OSCs based on non-halogenated NFAs with a bandgap below $1.28 \mathrm{eV}$. Our work suggests that the introduction of vinylene $\pi$-bridges is an alternative strategy to fine-tune the energy level and absorption range, and maintain planarity of the whole conjugated molecule, which endows these Y-series NFAs with the huge potential for constructing efficient OSCs based on ultra-NBG NFAs.

\section{Results and discussion}

\section{Synthesis}

The general synthetic routes of the four target Y-series NFAs are shown in Scheme S1 (ESI $\dagger$ ), and the detailed synthetic procedures for the intermediates can be found in the ESI. $\dagger$ Compound 3 was obtained in $80 \%$ yield through the Stille coupling reaction between 4,7-dibromo-5,6-dinitrobenzo[c] $]$ 1,2,5]thiadiazole (1) and tributyl(6-undecylthieno[3,2-b]thiophen-2-yl)stannane (2). The heptacyclic core 4 was obtained via a two-step successively the Cadogan reaction and the alkylation reaction from compound 3 in $45 \%$ yield. Compound $\mathbf{4}$ was then converted into its dialdehyde 5 via the Vilsmeier-Haack reaction in good yield. Subsequently, intermediate 5 was reacted with different doses of tributyl (1,3-dioxolan-2-ylmethyl)phosphonium bromide to afford precursors 6 and 7, respectively. The compound 7 was reacted with different doses of tributyl(1,3-dioxolan-2-ylmethyl)phosphonium bromide to produce compounds 8 and $\mathbf{9}$, respectively. The target four Y-series NFAs BTP-1V, BTP-2V, BTP-3V, and BTP-4V were obtained with good yields (82-85\%) through a typical Knoevenagel condensation between compounds 6, 7, 8, 9 and 1 , 1-dicyanomethylene-3-indanone. The ${ }^{1} \mathrm{H}$ and ${ }^{13} \mathrm{C}$ nuclear magnetic resonance (NMR) spectra and matrix-assisted laser desorption/ionization time of fight (MALDI-TOF) mass spectroscopy were employed to check all target compounds and molecules, which are summarized in the ESI. $\dagger$ The four target molecules exhibit good solubility in common organic solvents, such as dichloromethane, chloroform, and chlorobenzene, which is beneficial to solution processing of the photoelectric device. 


\section{Optical and electrochemical properties}

To better understand the effect of vinylene $\pi$-bridges on the optical properties of NFAs, the ultraviolet-visible (UV-Vis) absorption spectra of the four Y-series NFAs in dilute chloroform solution and in film are exhibited in Fig. 2. In dilute chloroform solutions, the absorption edges of these Y-series NFAs show a significantly red-shifted along with increasing numbers of vinylene units. These NFAs show strong and broad absorption in the range of 500-900 $\mathrm{nm}$. The maximum absorption values for these NFAs are observed at 737, 756, 768, $760 \mathrm{~nm}$ for BTP-1V, BTP-2V, BTP-3V, and BTP-4V, respectively. In films, all four Y-series NFAs exhibit obviously redshift absorption and vibration shoulder peaks in comparison to those of their solutions, which could be attributed to their strong intermolecular packing in the solid state. The absorption onsets in films of these NFAs are observed at 963, 968, 1020 and $1024 \mathrm{~nm}$, correspondingly their $E_{\mathrm{g}}^{\mathrm{opt}}$ of $1.28,1.28,1.22$ and $1.21 \mathrm{eV}$ for BTP-1V, BTP-2V, BTP-3V and BTP-4V, respectively. BTP-4V shows a very similar absorption profile with BTP-3V, indicating that further extending the length of $\pi$ conjugated backbone has little pronounced effect on broadening the NIR absorption. Compared with the absorption edge of Y6, a large bathochromic shift of 31-87 nm is observed for these BTP-Vbased Y-series NFAs, which indicates that vinylene $\pi$-bridges could gradually decrease the bandgap and broaden the absorption range. To the best of our knowledge, these BTP-V-based NFAs are the first example of Y-series NFAs attaching IC end group with a bandgap below $1.28 \mathrm{eV} .^{8,13,37-45}$ Generally, the fluorinated or chlorinated IC units are used as terminal groups for building efficient OSCs based on ultra-NBG NFAs. ${ }^{18,42-50}$ It should be noted that BTP-4V has an absorption onset of $1020 \mathrm{~nm}$, providing the smallest bandgaps (1.21 eV) among four ultra-NBG Y-series NFAs, despite attaching the nonhalogenated IC end group.

The highest occupied molecular orbital (HOMO) and lowest unoccupied molecular orbital (LUMO) energy levels of the four NFAs were measured by electrochemical cyclic voltammetry (CV) measurements with $\mathrm{Ag} / \mathrm{AgCl}$ as the reference electrode and the $\mathrm{Fc}^{+} / \mathrm{Fc}$ couple used as the internal standard at the scanning rate of $20 \mathrm{mV} \mathrm{s}^{-1}$, with $\mathrm{CV}$ curves shown in the ESI. $\dagger$ The onset oxidation/reduction potentials $\left(\varphi_{\text {ox/red }}\right)$ can be obtained from the cyclic voltammograms. According to the equations of $E_{\mathrm{HOMO} / \mathrm{LUMO}}=-e\left(\varphi_{\mathrm{ox} / \mathrm{red}}-\varphi_{\mathrm{Fc}^{+} / \mathrm{Fc}}+4.8\right)(\mathrm{eV})$, the $E_{\mathrm{HOMO}} / E_{\mathrm{LUMO}}$
Table 1 Optical and electrochemical properties of Y5, Y6 and four NFAs

\begin{tabular}{|c|c|c|c|c|c|c|}
\hline Acceptor & $\begin{array}{l}\lambda_{\max }^{a} \\
(\mathrm{~nm})\end{array}$ & $\begin{array}{l}\lambda_{\max }^{b} \\
(\mathrm{~nm})\end{array}$ & $\begin{array}{l}\lambda_{\text {onset }}{ }^{b} \\
\text { (nm) }\end{array}$ & $\begin{array}{l}E_{\mathrm{g}}^{\mathrm{opt} c} \\
(\mathrm{eV})\end{array}$ & $\begin{array}{l}\mathrm{HOMO}^{d} \\
(\mathrm{eV})\end{array}$ & $\begin{array}{l}\mathrm{LUMO}^{d} \\
(\mathrm{eV})\end{array}$ \\
\hline $\mathrm{Y}^{f}$ & 718 & 783 & 899 & 1.38 & $\begin{array}{l}-5.55 \\
(-5.74)^{e}\end{array}$ & $\begin{array}{l}-3.87 \\
(-3.70)^{e}\end{array}$ \\
\hline $\mathrm{Y} 6^{g}$ & 731 & 821 & 931 & 1.33 & $\begin{array}{l}-5.65 \\
(-)\end{array}$ & $\begin{array}{l}-4.10 \\
(-)\end{array}$ \\
\hline BTP-1V & 737 & 829 & 968 & 1.28 & $\begin{array}{l}-5.54 \\
(-5.69)^{e}\end{array}$ & $\begin{array}{l}-3.89 \\
(-3.70)^{e}\end{array}$ \\
\hline BTP-2V & 756 & 851 & 968 & 1.28 & $\begin{array}{l}-5.43 \\
(-5.64)^{e}\end{array}$ & $\begin{array}{l}-3.91 \\
(-3.70)^{e}\end{array}$ \\
\hline BTP-3V & 768 & 814 & 1018 & 1.22 & $\begin{array}{l}-5.38 \\
(-5.58)^{e}\end{array}$ & $\begin{array}{l}-3.90 \\
(-3.70)^{e}\end{array}$ \\
\hline BTP-4V & 760 & 885 & 1021 & 1.21 & $\begin{array}{l}-5.34 \\
(-5.52)^{e}\end{array}$ & $\begin{array}{l}-3.94 \\
(-3.70)^{e}\end{array}$ \\
\hline
\end{tabular}

${ }^{a}$ In chloroform solutions. ${ }^{b}$ In neat films. ${ }^{c} E_{\mathrm{g}}^{\mathrm{opt}}=1240 / \lambda_{\text {onset. }}{ }^{d}$ Calculated according to the equation $E_{\mathrm{LUMO} / \mathrm{HOMO}}=-e\left(E_{\mathrm{red} / \mathrm{ox}}+4.44\right)$. ${ }^{e}$ Obtained from DFT calculations. ${ }^{f}$ Ref. $34{ }^{g}$ Ref. 8

values of BTP-1V, BTP-2V, BTP-3V, and BTP-4V are calculated to be $-5.54 /-3.89,-5.43 /-3.91,-5.38 /-3.90$, and $-5.34 /-3.94 \mathrm{eV}$, respectively. The electrochemical data of BTP-V-based NFAs are summarized in Table 1. Having obtained the energy level of these four Y-series NFAs, we then compare them in an energy diagram displayed in Fig. 2c. It is worth noting that extending the $\pi$-conjugated length of the molecular backbone obviously elevates the HOMO energy levels of the BTP-V-based NFAs, and meanwhile slightly lowers the LUMO energy levels, where it effectively reduces the bandgap of these NFAs due to the electron-donating properties of the vinylene $\pi$-bridge. Compared with the HOMO/LUMO value of Y5 $(-5.55 /-3.87 \mathrm{eV}){ }^{34}$ the HOMO energy levels of the BTP-V-based NFAs are gradually upshifted and their LUMO energy levels are downshifted slightly. The LUMO levels of these NFAs are also very close to that of the electron transport layer, $[(9,9-b i s$ (3'-( $N, N$-dimethylamino)propyl)-2,7-fluorene)-alt-5, $5^{\prime}$-bis(2,2' -thiophene)-2,6-naphthalene-1,4,5,8-tetracaboxylic- $N, N^{\prime}$-di(2-ethylhexyl) imide] (PNDIT-F3N), ${ }^{46}$ hence promoting efficient charge collection. The result demonstrates that the vinylene $\pi$-bridge is an effective method to adjust the bandgaps and energy levels of NFAs.

\section{Theoretical calculation}

For rational design of ultra-NBG NFAs, the density functional theory (DFT) calculations were employed to screen possible candidates of the Y-series NFAs with a rigid backbone, and (a)

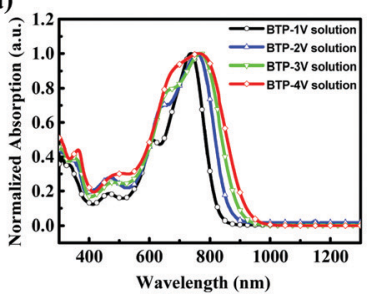

(b)

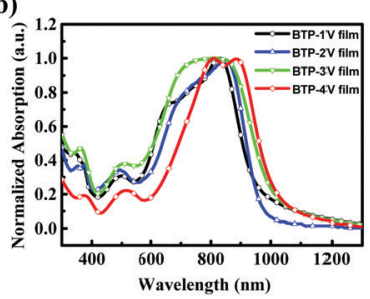

(c)

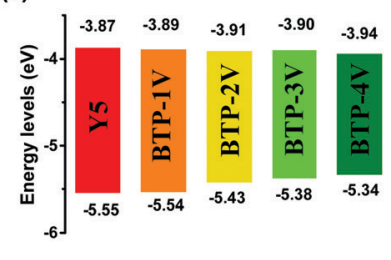

(d)

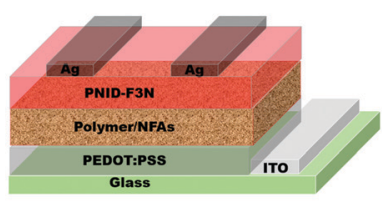

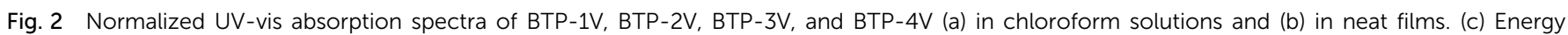
diagrams of $\mathrm{Y} 5, \mathrm{BTP}-1 \mathrm{~V}, \mathrm{BTP}-2 \mathrm{~V}, \mathrm{BTP}-3 \mathrm{~V}$ and BTP-4V. (d) Architecture of the BTP-V-based devices. 

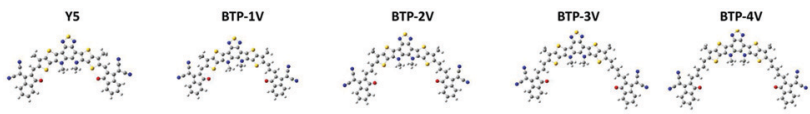
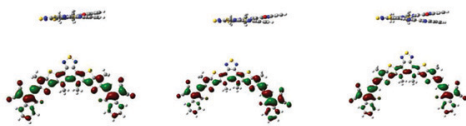

LUMO $=-3.7002 \mathrm{eV}$

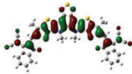

LUMO $=-3.7049 \mathrm{e}$
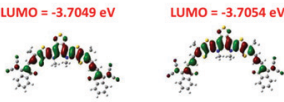

HоMо $=-5.6410 \mathrm{e}$
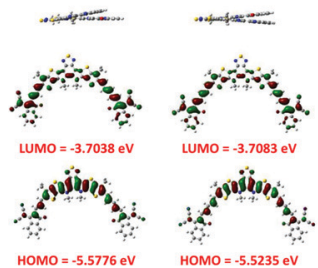

LUMO $=-3.7083 \mathrm{eV}$

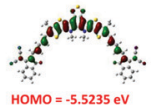

Fig. 3 The optimized conformation of Y5, BTP-1V, BTP-2V, BTP-3V and BTP-4V by standard DFT calculations.

proper HOMO/LUMO energy levels. Compared with the multifused ring on the central core to enhance electron-donating ability, the increasing conjugation length of Y5 was adopted to narrow its bandgap. The DFT calculations of Y5, BTP-1V, BTP-2V, BTP-3V and BTP-4V were performed with Gaussian at the B3LYP/6-31G(d,p) level to elucidate the influence of inserting vinylene onto the backbone of the fused conjugated molecule on molecular geometries and energy level of molecules, where the long alkyl side chains were simplified into methyl groups (Fig. 3). Seen from optimized conformation of five molecules, similar distributions of HOMOs and LUMOs were seen from Y5 to four BTP-V-based NFAs. The acceptor consists of two planar units, with a twist in the nitrogen atom of the central core attached alkyl groups. The dihedral angles of $\mathrm{N}-\mathrm{C}-$ $\mathrm{C}-\mathrm{N}$ were 10.1, 10.0, 9.9, 9.9 and $9.9^{\circ}$ for Y5, BTP-1V, BTP-2V, BTP-3V and BTP-4V, respectively, indicating perfect coplanarity for these four Y-series NFAs. The conjugated skeleton length of these NFAs was designed to have different amounts of vinylene $\pi$-bridges, with the aiming to subtly regulate the conjugation length of the acceptor while promoting the $\pi$-electron delocalization, and thereby facilitating photon absorption and charge transportation in the solid state. The intramolecular $\mathrm{H} \cdots \mathrm{O}$ conformational lock offered four NFAs a planar structure to promote $\pi-\pi$ stacking in the solid state. The HOMO/LUMO energy levels of Y5, BTP-1V, BTP-2V, BTP-3V and BTP-4V were calculated to be $-5.74 /-3.70,-5.69 /-3.70,-5.64 /-3.70$, $-5.58 /-3.70$, and $-5.52 /-3.70 \mathrm{eV}$, respectively, which were consistent with the result of $\mathrm{CV}$ measurements and $\mathrm{UV}$-vis spectra. An increase in the $\pi$-conjugated length of the backbone of the Y-series NFAs clearly upshifted the HOMO level and basically maintained the LUMO level. According to theory calculation, the HOMO orbital was mainly located at the electron-donating core, while the LUMO orbital was delocalized across the entire molecule with more focus on the electronwithdrawing end groups, facilitating charge transport within and across molecules.

\section{Photovoltaic properties}

To investigate the photovoltaic performance of the four Y-series NFAs, single-junction OSCs with a conventional architecture of ITO/PEDOT:PSS/active layer/PNDIT-F3N/Ag (Fig. 2d) were fabricated. The details of device photovoltaic parameters are listed in Table 2. The PEDOT:PSS and PNDIT-F3N ${ }^{46}$ were selected as the hole and electron transport layer, respectively. Considering matched energy and complementary absorption, the polymer PBDB-T was selected as the donor material for BTP-1V (PCE10 for BTP-2V, BTP-3V and BTP-4V). The details of device preparation can be found in the ESI. $\dagger$ The current density-voltage $(J-V)$ curves of the optimal BTP-V-based OSC devices are plotted in Fig. 4a, and the detailed photovoltaic parameters are summarized in Table 2. The PCE10/BTP-4V- and PCE10/BTP-3V-based device showed the low PCE of $1-2 \%$, where the values of $J_{\mathrm{sc}}\left(3.61 \mathrm{~mA} \mathrm{~cm}{ }^{-2}\right.$ for BTP$4 \mathrm{~V}, 6.46 \mathrm{~mA} \mathrm{~cm}^{-2}$ for BTP-3V), $V_{\mathrm{oc}}(0.67 \mathrm{~V}$ for BTP-4V, $0.72 \mathrm{~V}$ for BTP-3V) and FF (0.42 for BTP-4V, 0.44 for BTP-3V) are very poor. When the number of the vinyl $\pi$-bridges of the BTP-V-based NFAs gradually reducing, the photovoltaic parameters of OSCs dramatically increased. Compared with the PCE10/BTP-3V-based device, the BTP-2V-based device exhibited a much higher PCE of 7.78\% due to the increased $J_{\mathrm{sc}}\left(19.80 \mathrm{~mA} \mathrm{~cm}{ }^{-2}\right), V_{\mathrm{oc}}(0.77 \mathrm{~V})$, and $\mathrm{FF}$ (0.51). Meanwhile, changing PCE10 to PBDB-T, the PBDB/BTP-1Vbased device showed a slightly enhanced $J_{\mathrm{sc}}\left(20.86 \mathrm{~mA} \mathrm{~cm}{ }^{-2}\right)$, significantly increased $V_{\mathrm{oc}}(0.84 \mathrm{~V})$ and $\mathrm{FF}(0.63)$, and thus a highest PCE of $11.03 \%$. To our knowledge, the PCE of $11 \%$ is the highest value for binary single-junction OSCs based on ultra-NBG $\left(E_{\mathrm{g}} \leq 1.28 \mathrm{eV}\right)$ non-halogenated NFAs with IC ending groups to date. $^{34,47-50}$ The values of $V_{\mathrm{oc}}$ are decreased from BTP-1V $(0.84 \mathrm{~V})$, BTP-2V $(0.77 \mathrm{~V})$, BTP-3V $(0.72 \mathrm{~V})$ to BTP-4V $(0.67 \mathrm{~V})$, which is consistent with energy level alignment.

The current densities of all best-performance devices were calibrated with the corresponding EQE data. The EQE spectra of the four BTP-V-based devices are shown in Fig. 4b. The integrated $J_{\mathrm{sc}}$ values from EQE curves of PBDB-T/BTP-1V- and PCE10/BTP-2V-based devices are 20.53 and $18.92 \mathrm{~mA} \mathrm{~cm}$, respectively, matching well with the $J_{\text {sc }}$ values from the $J-V$ curves (less than 5\% mismatch). Generally, the PCE10/BTP-2Vbased device exhibits a more broadened EQE response from 300 to $960 \mathrm{~nm}$ compared with that of the device based on PBDB-T/BTP-1V. This result is consistent with their UV-visible NIR absorption spectra in thin film. However, the PBDB-T/BTP$1 \mathrm{~V}$-based device showed much higher average EQE values

Table 2 Photovoltaic parameters of devices based on four Y-series NFAs

\begin{tabular}{|c|c|c|c|c|c|c|}
\hline Materials & $V_{\mathrm{oc}}{ }^{a}(\mathrm{~V})$ & $J_{\mathrm{sc}}{ }^{a}\left(\mathrm{~mA} \mathrm{~cm}^{-2}\right)$ & $J_{\mathrm{sc}}{ }^{b}\left(\mathrm{~mA} \mathrm{~cm}^{-2}\right)$ & $\mathrm{FF}^{a}(\%)$ & PCE (\%) & $\mu_{\mathrm{h}} / \mu_{\mathrm{e}}\left(10^{-4} \mathrm{~cm}^{2} \mathrm{~V}^{-1} \mathrm{~s}^{-1}\right)$ \\
\hline PBDB-T/BTP-1V & $0.82 \pm 0.01(0.84)$ & $20.58 \pm 0.30(20.86)$ & 20.53 & $62.3 \pm 0.7(63.1)$ & $10.70 \pm 0.3(11.03)$ & $4.48 / 4.80$ \\
\hline PCE10/BTP-2V & $0.75 \pm 0.02(0.77)$ & $19.42 \pm 0.25(19.80)$ & 18.92 & $51.0 \pm 0.4(51.4)$ & $7.41 \pm 0.25(7.87)$ & $6.85 / 2.66$ \\
\hline PCE10/BTP-3V & $0.70 \pm 0.02(0.72)$ & $6.34 \pm 0.09(6.46)$ & 4.68 & $43.9 \pm 0.3(44.3)$ & $1.95 \pm 0.07(2.06)$ & $9.68 / 1.15$ \\
\hline PCE10/BTP-4V & $0.65 \pm 0.01(0.67)$ & $3.54 \pm 0.50(3.61)$ & 1.24 & $42.1 \pm 0.4(42.7)$ & $0.97 \pm 0.03(1.03)$ & $16.65 / 2.64$ \\
\hline
\end{tabular}

${ }^{a}$ In parentheses are the average values of 10 devices. ${ }^{b}$ Obtained from the integration of EQE spectra. 
(a)

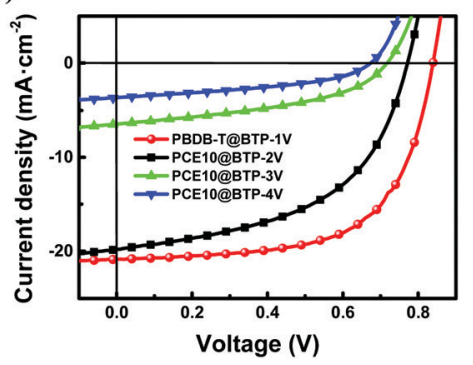

(b)

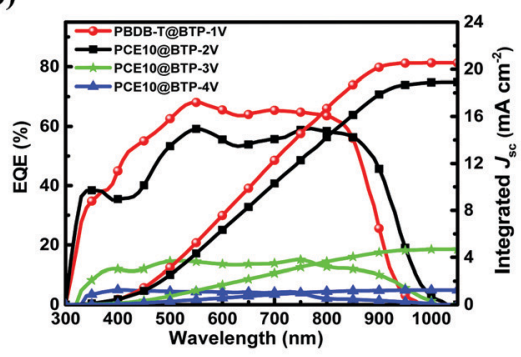

(c)

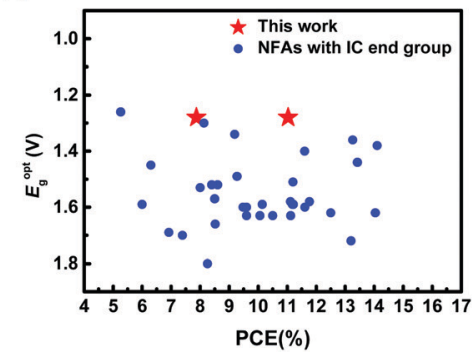

Fig. 4 (a) J-V characteristics, (b) external quantum efficiency (EQE) spectra of NFAs-based devices. (c) A summary of the reported binary single-junction OSCs based on non-halogenated NFAs with IC end groups.

compared with those of the PCE10/BTP-2V-based device. The better stacking in PBDB-T/BTP-1V films is proposed to be the reason for the higher EQE despite their narrower absorption range. A discussion on film morphologies will be covered in later sections to support this point. The higher average EQE value is the main reason for the improved $J_{\mathrm{sc}}$ value for the PBDB-T/BTP-1V-based device. As a result, in combination with a large $V_{\mathrm{oc}}$ and $J_{\mathrm{sc}}$, the PBDB-T/BTP-1V-based device exhibited a much higher PCE of $11.03 \%$ compared with that of the PCE10/ BTP-2V-based device. The integrated currents from the EQE spectra of PCE10/BTP-3V- and PCE10/BTP-4V-based devices are relatively low due to their inefficiently harvesting photons in a broad absorption range.

It is noteworthy that the corresponding energy loss $\left(E_{\text {loss }}\right)$ for the BTP-1V-based device is calculated to be $0.58 \mathrm{eV}$. Generally, $E_{\text {loss }}$ is defined by $E_{\text {loss }}=E_{\mathrm{g}}-q V_{\text {oc }}$, where $E_{\mathrm{g}}$ refers to the optical bandgap determined by the smaller value of a donor or an acceptor. ${ }^{51,52}$ It is a relatively small value among the NIR absorption Y-series NFAs. ${ }^{8,19,53}$ The value of $E_{\text {loss }}$ is important to overcome the trade-off between $V_{\mathrm{oc}}$ and $J_{\mathrm{sc}}$, which could boost the two parameters simultaneously for high efficiency NFA-based OSCs. Meanwhile, such a small $E_{\text {loss }}$ of the BTP-1Vbased device is achieved from the Y-series NFAs with an $E_{\mathrm{g}}^{\text {opt }}$ of $1.28 \mathrm{eV}$, indicating a brighter future of efficient OSCs based on ultra-NBG Y-series NFAs.

\section{Charge recombination and carrier mobility}

The exciton dissociation and charge extraction behaviours of the respective devices were further studied by plotting photogenerated current density $\left(J_{\mathrm{ph}}\right)$ against effective voltage $\left(V_{\text {eff }}\right)$ (Fig. S27, ESI $\dagger$ ). $J_{\mathrm{ph}}$ is defined as $J_{\mathrm{L}}-J_{\mathrm{D}}, V_{\text {eff }}$ is calculated as $V_{\mathrm{o}}-V_{\mathrm{a}}$, where $J_{\mathrm{L}}$ and $J_{\mathrm{D}}$ represent the current densities under the illumination of AM $1.5 \mathrm{G} 100 \mathrm{~mW} \mathrm{~cm}^{-2}$ and in the dark, $V_{\mathrm{o}}$ is the voltage when $J_{\mathrm{ph}}$ is zero and $V_{\mathrm{a}}$ is the voltage applied voltage externally. The value of $J_{\mathrm{ph}} / J_{\mathrm{sat}}$ (where $J_{\mathrm{sat}}$ is saturation photo-generated current density) is defined as a charge exciton dissociation probability $\left(P_{\text {diss }}\right)$. The BTP-1V-based device exhibited the highest $P_{\text {diss }}$ value $(93.9 \%)$ in the four BTP-V-based devices $(82.3 \%$ for the BTP-2V-based device, $53.4 \%$ for the BTP-3V-based device, and $50.8 \%$ for the BTP-4V-based device). This implies the most efficient charge extraction and charge collection in the BTP-1V-based device among the four BTP-Vbased devices.
The light intensity $\left(P_{\text {light }}\right)$ dependence of $J_{\text {sc }}$ was determined to evaluate the charge carrier recombination behaviour of these four BTP-V-based devices. The plots of $\log J_{\mathrm{sc}}$ versus $\log P_{\text {light }}$ are shown in Fig. S28 (ESI $\dagger$ ). Theoretically, the correlation between $J_{\mathrm{sc}}$ and $P_{\text {light }}^{\alpha}$ is written as $J_{\mathrm{sc}} \propto P_{\text {light }}^{\alpha}$, where $\alpha$ is the bimolecular recombination index. ${ }^{54}$ When the $\alpha$ value gets close to 1 , it implies that all dissociated charges are collected with minimal bimolecular recombination. In our cases, the $\alpha$ values are 0.93, $0.86,0.83$ and 0.85 for BTP-1V-, BTP-2V-, BTP-3V- and BTP-4Vbased devices, respectively, indicating the most effective carrier collection and the weakest bimolecular recombination in BTP1V-based devices, thus leading to the highest FF. Fig. S29 (ESI $\dagger$ ) shows the plots of $V_{\text {oc }}$ versus $\ln P_{\text {light }}$ of the four optimized BTP-V-based OSC devices. For bimolecular recombination in BHJ OSCs, the semilogarithmic plot of $V_{\text {oc }}$ as a function of the light intensity should show a linear relationship with a slope of $1 \times k T / q$, where $k$ is the Boltzmann constant, $T$ is room temperature $(298 \mathrm{~K})$, and $q$ is the elementary charge. ${ }^{55}$ In contrast, a slope of $2 \times k T / q$ implies that the trap-assisted or monomolecular recombination is the dominating mechanism. ${ }^{56}$ The slopes of the four BTP-V-based device curves represent $1.20 \mathrm{kT} / \mathrm{q}, 1.71 \mathrm{kT} / \mathrm{q}, 2.53 \mathrm{kT} / \mathrm{q}$ and $4.01 \mathrm{kT} / \mathrm{q}$ for BTP-1V-, BTP-2V-, BTP-3V- and BTP-4V-based devices respectively, signifying the dominant bimolecular recombination for BTP-1Vbased devices, whereas BTP-2V-, BTP-3V- and BTP-4V-based devices were affected by monomolecular or trap-assisted recombination. The results collectively suggest that charge recombination is effectively suppressed in the BTP-1V-based device. The existence of monomolecular or trap-assisted recombination indicates that domain sizes in the PCE10/BTP-2V, PCE10/ BTP-3V and PCE10/BTP-4V blends are large to some extent, whereas the PBDB-T/BTP-1V blends may have more appropriate domain sizes.

Additionally, the photoluminescence (PL) measurements of the neat and blend films were carried out to investigate the exciton dissociation and photo-induced charge transfer between polymer donors and Y-series NFAs. As shown in Fig. S25 (ESI $\dagger$ ), PL intensities of the pure acceptors decrease dramatically upon incorporating other components in the corresponding blend films which suggests that the exciton dissociation and charge transfer between polymers and the four acceptors are generally efficient. PBDB-T/BTP-1V and PCE10/BTP-2V blend films exhibit sufficiently quenching 
efficiencies (91\% for BTP-1V and $87 \%$ for BTP-2V) in PL experiments, implying efficient exciton dissociation and charge transfer in blend layers. Meanwhile, PCE10/BTP-3V and PCE10/BTP-4V blend films exhibit deficiently quenching efficiencies $(71 \%$ for BTP-3V and 66\% for BTP-4V) in PL quenching experiments, which indicates that less free charge can be generated in the blends and results in a low external quantum efficiency (EQE) of BTP-3V- and BTP-4V-based devices. It is clear that PBDB-T/BTP-1V exhibits the most efficient hole and electron transfer while PCE10/BTP-4V shows the worst, which is consistent with their different $J_{\mathrm{sc}}$ and EQE response in the corresponding devices.

The space-charge-limited current (SCLC) method was utilized to characterize the charge transport properties of the blend films, as charge transport is closely relevant to molecular packing. Experiments were carried out on the hole-only and electron-only devices with the structures of ITO/PEDOT:PSS/blend film/ $\mathrm{MoO}_{3} / \mathrm{Al}$ and ITO/ZnO/blend film/PNDITF3N/Al, respectively. We measured the charge carrier mobilities by fitting the dark $J-V$ curves of these devices. The electron mobility $\left(\mu_{\mathrm{e}}\right)$ and the hole mobility $\left(\mu_{\mathrm{h}}\right)$ are summarized in Table 2, and the $J-V$ curves under the dark condition are shown in Fig. S30 (ESI $\dagger$ ). The values of $\mu_{\mathrm{h}} / \mu_{\mathrm{e}}$ are $4.48 \times 10^{-4} / 4.80 \times 10^{-4}$, $6.85 \times 10^{-4} / 2.66 \times 10^{-4}, 9.68 \times 10^{-4} / 1.15 \times 10^{-4}, 16.45 \times 10^{-4} /$ $2.64 \times 10^{-4} \mathrm{~cm}^{2} \mathrm{~V}^{-1} \mathrm{~s}^{-1}$ for PBDB-T/BTP-1V, PCE10/BTP-2V, PCE10/BTP-3V and PCE10/BTP-4V blend films, respectively. Compared with PCE10/BTP-2V, PCE10/BTP-3V and PCE10/BTP-4V, the PBDB-T/BTP-1V blend film shows the most balanced $\mu_{\mathrm{h}} / \mu_{\mathrm{e}}$ ratio of 0.93, which is beneficial for charge transport and collection, thus leading to the high $J_{\mathrm{sc}}$ and $\mathrm{FF}$ in the corresponding devices, whereas the unbalanced mobilities of PCE10/BTP-3V and PCE10/BTP-4V blend films are the main reasons for the inferior values of $J_{\mathrm{sc}}$ and $\mathrm{FF}$.

\section{Morphology}

Atomic force microscopy (AFM) was utilized to investigate the surface morphology of active layers (Fig. 5). In AFM height images, all four blends show smooth surface morphologies, and the root mean square roughness $\left(R_{\mathrm{q}}\right)$ values of PBDB-T/BTP-1V, PCE10/ BTP-2V, PCE10/BTP-3V and PCE10/BTP-4V blend films are 1.27, $1.19,0.94$ and $0.81 \mathrm{~nm}$, respectively. As shown in AFM phase images, in compassion to the BTP-2V, BTP-3V and BTP-4V-based blends, the BTP-1V-based blend exhibits the smallest phase separation, which is beneficial to construct bi-continuous interpenetrating networks in the blend film. The PBDB-T/BTP-1V blend film displays a more well-ordered morphology of BTP-1V and PBDB-T interlayers in the blend film, which facilitates the efficient exciton dissociation and charge transport to yield better $J_{\mathrm{sc}}$ and $\mathrm{FF}$ in the related devices.

To investigate the molecular stacking and crystallization of the four BTP-V-based blend films, two-dimensional grazing incidence wide-angle X-ray scattering (GIWAXS) measurements were performed on pure and blend films. As shown in Fig. 6, the 2D GIWAXS patterns and the corresponding 1D line-cuts in the corresponding 1D line-cuts in the out-of-plane (OOP) and in-plane (IP) directions of the four Y-series NFAs suggest that four blend films adopt a preferential face-on orientation with respect to the substrate, as indicated by the strong (010) $\pi-\pi$ stacking peaks in the OOP direction and the (100) lamellar stacking peaks in the IP direction, which is beneficial for charge transport in the vertical direction across the blend films. The charge trend of $\pi-\pi$ stacking distances of the four blends is similar to that of the corresponding neat acceptor films. The PBDB-T/BTP-1V, PCE10/BTP-2V, PCE10/BTP-3V, and PCE10/ BTP-4V blend films showed obvious (010) diffraction peaks at the $q_{z}$ of $1.73 \AA^{-1}, 1.71 \AA^{-1}, 1.72 \AA^{-1}$, and $1.70 \AA^{-1}$, respectively,
PBDB-T/BTP-1V
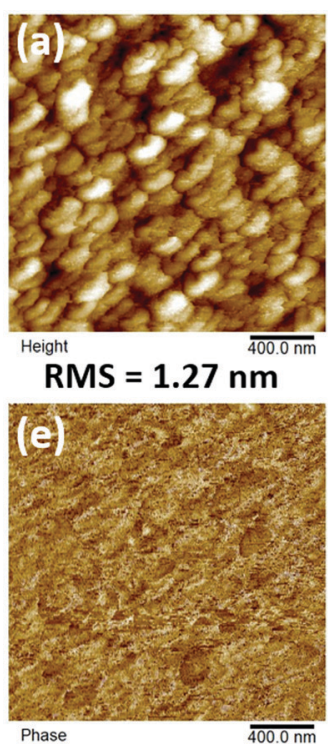

\section{PCE10/BTP-2V}

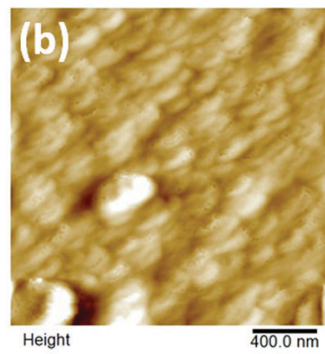

$\mathrm{RMS}=1.19 \mathrm{~nm}$

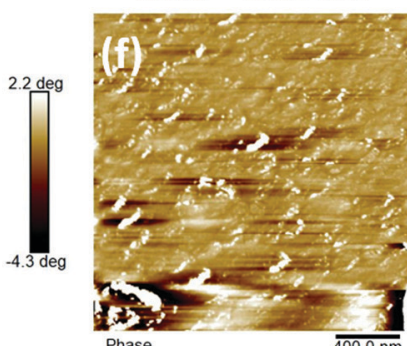

PCE10/BTP-3V

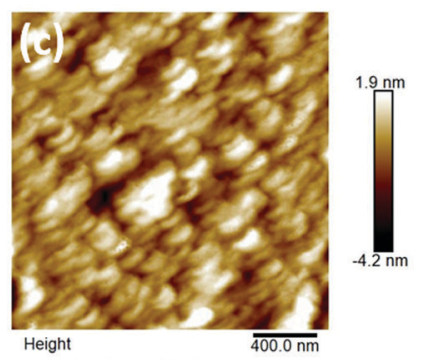

RMS $=0.94 \mathrm{~nm}$

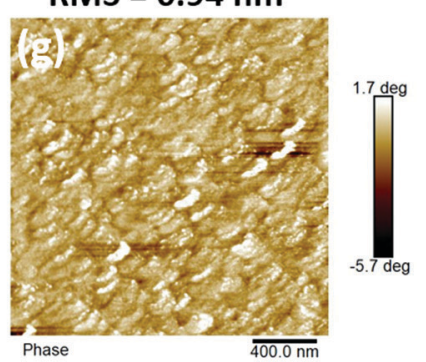

PCE10/BTP-4V

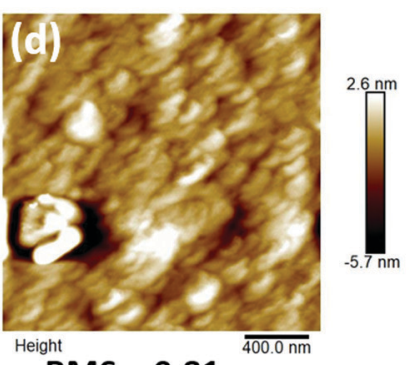

RMS $=0.81 \mathrm{~nm}$

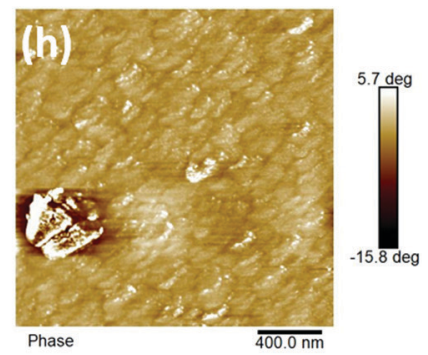

Fig. 5 AFM height (a-d) and AFM phase (e-h) of the PBDB-T/BTP-1V, PCE10/BTP-2V, PCE10/BTP-3V, and PCE10/BTP-4V blend films. 

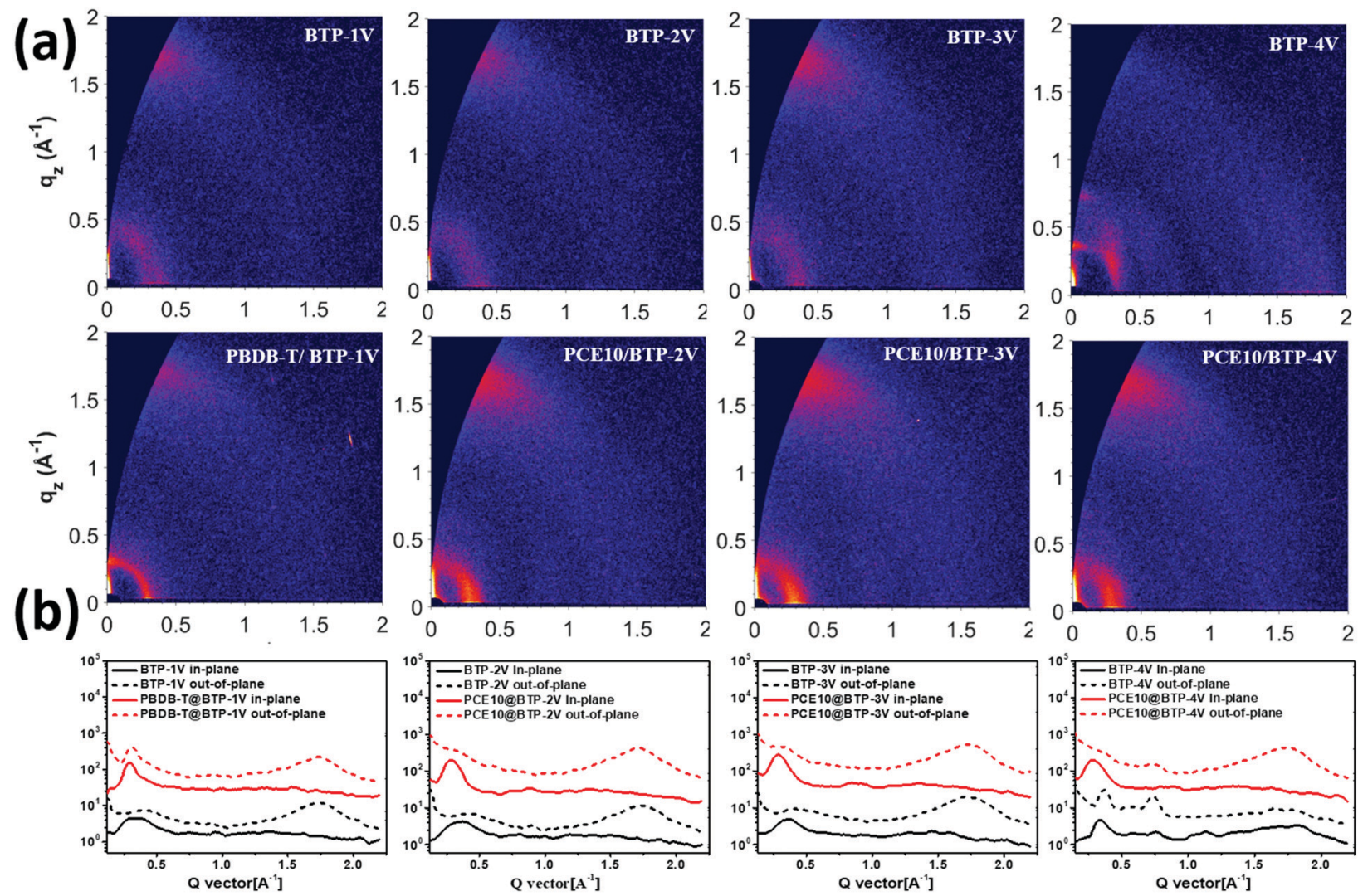

Fig. 6 (a) GIWAXS patterns of neat and blend films. (b) Corresponding 1D-line-cut profiles of GIWAXS patterns for four blend films.

and the corresponding $\pi-\pi$ stacking distances are calculated to be $3.63 \AA$, $3.67 \AA$, $3.65 \AA$ and $3.69 \AA$. The PBDB-T/BTP-1V blend film exhibits the shortest $\pi-\pi$ stacking distance in the four blend films. It is envisaged that the closest $\pi-\pi$ stacking distance of the PBDB-T/BTP-1V blend film facilitates the charge transport and restrains charge recombination, thus accounting for the best $J_{\mathrm{sc}}, \mathrm{FF}$ and PCE values of PBDB-T/BTP-1V-based devices. The GIWAXS results are generally consistent with the results of charge recombination studies.

\section{Conclusions}

In summary, we initially designed and synthesized a series of the Y-series BTP-V-based NFAs through a general versatile synthetic approach, featuring ultra-NBG and various electron accepting abilities. Compared with side chain and terminal group engineering, this synthetic approach of the Y-series NFAs simplified the extending conjugated backbone of NFAs by introducing vinylene $\pi$-bridges. More importantly, this work opens the door to a great variety of Y6 family. These changes of molecular structures have a huge influence on the optical, electronic, and photovoltaic properties of these related BTP-Vbased NFAs. The PBDB-T/BTP-1V blend film shows the highest electron mobility, the best balance charge transport, and the least charge recombination among four BTP-V-based NFAs. To the best of our knowledge, this is the first time that the PCE of over $11 \%$ has been realized using a non-halogenated Y-series
NFA with a bandgap below $1.28 \mathrm{eV}$. The result not only demonstrates that the fine-tuning the extent of NFAs conjugated system is an effective method to adjust their energy levels, optical absorption, charge transport, and morphology, but also provides a useful guideline to alter the electrochemical properties of the Y-series NFAs for high-performance ultra-NBG NFAs-based OSCs.

\section{Author contributions}

J. H. synthesized the four non-fullerene acceptors. S. L. carried out the materials selection, cells fabrication and device characterizations. $\mathrm{H}$. Y. carried out the UV-vis, fluorescence, TGA and GIWAXS measurements. H. C. conducted the mass spectrometry and cyclic voltammetry measurements. Z. L. carried out $\mathrm{H}^{1}$ NMR. J. H., S. L., H. Y., L. L., Y. Z., and H. Y. prepared the manuscript and all authors commented on the manuscript. L. L. and H. Y. directed the project.

\section{Conflicts of interest}

The authors declare that they have no known competing financial interests or personal relationships that could have appeared to influence the work reported in this paper. 


\section{Acknowledgements}

The work described in this paper was supported by the Basic and Applied Basic Research Major Program of Guangdong Province (No. 2019B030302007), the Natural Science Foundation of China (Grant No. 21962005), the Natural Science Foundation of Guangxi Province (Grant No. 2017GXNSFAA198343 and 2018 GXNSFAA281137) and the Key Laboratory Research Fund of Guangxi Province (EMFM20161101).

\section{References}

1 G. Zhang, J. Zhao, P. C. Y. Chow, K. Jiang, J. Zhang, Z. Zhu, J. Zhang, F. Huang and H. Yan, Chem. Rev., 2018, 118, 3447-3507.

2 J. Zhang, H. S. Tan, X. Guo, A. Facchetti and H. Yan, Nat. Energy, 2018, 3, 720-731.

3 M. Li, K. Gao, X. Wan, Q. Zhang, B. Kan, R. Xia, F. Liu, X. Yang, H. Feng, W. Ni, Y. Wang, J. Peng, H. Zhang, Z. Liang, H.-L. Yip, X. Peng, Y. Cao and Y. Chen, Nat. Photonics, 2017, 11, 85-90.

4 E. M. Speller, A. J. Clarke, J. Luke, H. K. H. Lee, J. R. Durrant, N. Li, T. Wang, H. C. Wong, J.-S. Kim, W. C. Tsoi and Z. Li, J. Mater. Chem. A, 2019, 7, 23361-23377.

5 J. Liu, S. Chen, D. Qian, B. Gautam, G. Yang, J. Zhao, J. Bergqvist, F. Zhang, W. Ma, H. Ade, O. Inganäs, K. Gundogdu, F. Gao and H. Yan, Nat. Energy, 2016, 1, 16089.

6 Y. Lin, J. Wang, Z.-G. Zhang, H. Bai, Y. Li, D. Zhu and X. Zhan, Adv. Mater., 2015, 27, 1170-1174.

7 T. Liu, Z. Luo, Y. Chen, T. Yang, Y. Xiao, G. Zhang, R. Ma, X. Lu, C. Zhan, M. Zhang, C. Yang, Y. Li, J. Yao and H. Yan, Energy Environ. Sci., 2019, 12, 2529-2536.

8 J. Yuan, Y. Zhang, L. Zhou, G. Zhang, H.-L. Yip, T.-K. Lau, X. Lu, C. Zhu, H. Peng, P. A. Johnson, M. Leclerc, Y. Cao, J. Ulanski, Y. Li and Y. Zou, Joule, 2019, 3, 1140-1151.

9 Q. Liu, Y. Jiang, K. Jin, J. Qin, J. Xu, W. Li, J. Xiong, J. Liu, Z. Xiao, K. Sun, S. Yang, X. Zhang and L. Ding, Sci. Bull., 2020, 65, 272-275.

10 Y. Cui, H. Yao, J. Zhang, K. Xian, T. Zhang, L. Hong, Y. Wang, Y. Xu, K. Ma, C. An, C. He, Z. Wei, F. Gao and J. Hou, Adv. Mater., 2020, 32, 1908205.

11 L. Liu, Y. Kan, K. Gao, J. Wang, M. Zhao, H. Chen, C. Zhao, T. Jiu, A.-K.-Y. Jen and Y. Li, Adv. Mater., 2020, 32, 1907604.

12 R. Ma, T. Liu, Z. Luo, Q. Guo, Y. Xiao, Y. Chen, X. Li, S. Luo, X. Lu, M. Zhang, Y. Li and H. Yan, Sci. China: Chem., 2019, 63, 325-330.

13 Y. Cui, H. Yao, L. Hong, T. Zhang, Y. Tang, B. Lin, K. Xian, B. Gao, C. An, P. Bi, W. Ma and J. Hou, Natl. Sci. Rev., 2019, 7, 1239-1246.

14 J. Gao, W. Gao, X. Ma, Z. Hu, C. Xu, X. Wang, Q. An, C. Yang, X. Zhang and F. Zhang, Energy Environ. Sci., 2020, 13, 958-967.

15 F. Cai, C. Zhu, J. Yuan, Z. Li, L. Meng, W. Liu, H. Peng, L. Jiang, Y. Li and Y. Zou, Chem. Commun., 2020, 56, 4340-4343.
16 F. Cai, H. Peng, H. Chen, J. Yuan, J. Hai, T.-K. Lau, J. Wang, Y. Hu, W. Liu, X. Lu and Y. Zou, J. Mater. Chem. A, 2020, 8, 15984-15991.

17 Y. Chen, F. Bai, Z. Peng, L. Zhu, J. Zhang, X. Zou, Y. Qin, H. K. Kim, J. Yuan, L.-K. Ma, J. Zhang, H. Yu, P. C. Y. Chow, F. Huang, Y. Zou, H. Ade, F. Liu and H. Yan, Adv. Energy Mater., 2020, 2003141.

18 H. Yu, Z. Qi, J. Zhang, Z. Wang, R. Sun, Y. Chang, H. Sun, W. Zhou, J. Min, H. Ade and H. Yan, J. Mater. Chem. A, 2020, 8, 23756-23765.

19 F. Qi, K. Jiang, F. Lin, Z. Wu, H. Zhang, W. Gao, Y. Li, Z. Cai, H. Y. Woo, Z. Zhu and A. K. Y. Jen, ACS Energy Lett., 2020, 6, 9-15.

20 H. Yao, Y. Cui, R. Yu, B. Gao, H. Zhang and J. Hou, Angew. Chem., Int. Ed., 2017, 56, 3045-3049.

21 S. Chen, H. Yao, B. Hu, G. Zhang, L. Arunagiri, L.-K. Ma, J. Huang, J. Zhang, Z. Zhu, F. Bai, W. Ma and H. Yan, Adv. Energy Mater., 2018, 8, 1800529.

22 D. Liu, B. Kan, X. Ke, N. Zheng, Z. Xie, D. Lu and Y. Liu, Adv. Energy Mater., 2018, 8, 1801618.

23 S. Yu, Y. Chen, L. Yang, P. Ye, J. Wu, J. Yu, S. Zhang, Y. Gao and H. Huang, J. Mater. Chem. A, 2017, 5, 21674-21678.

24 Y. Liu, Z. Zhang, S. Feng, M. Li, L. Wu, R. Hou, X. Xu, X. Chen and Z. Bo, J. Am. Chem. Soc., 2017, 139, 3356-3359.

25 Y.-Q.-Q. Yi, H. Feng, X. Ke, J. Yan, M. Chang, X. Wan, C. Li and Y. Chen, J. Mater. Chem. C, 2019, 7, 4013-4019.

26 H. Wang, J. Cao, J. Yu, Z. Zhang, R. Geng, L. Yang and W. Tang, J. Mater. Chem. A, 2019, 7, 4313-4333.

27 Y. R. Cheon, Y. J. Kim, J.-J. Ha, M.-J. Kim, C. E. Park and Y.-H. Kim, Macromolecules, 2014, 47, 8570-8577.

28 E. H. Jung and W. H. Jo, Energy Environ. Sci., 2014, 7, 650-654.

29 S. Fukuta, J. Seo, H. Lee, H. Kim, Y. Kim, M. Ree and T. Higashihara, Macromolecules, 2017, 50, 891-899.

30 Y. Guo, Y. Li, O. Awartani, H. Han, J. Zhao, H. Ade, H. Yan and D. Zhao, Adv. Mater., 2017, 29, 1700309.

31 X. Li, H. Huang, H. Bin, Z. Peng, C. Zhu, L. Xue, Z.-G. Zhang, Z. Zhang, H. Ade and Y. Li, Chem. Mater., 2017, 29, 10130-10138.

32 H. Huang, X. Li, L. Zhong, B. Qiu, Y. Yang, Z.-G. Zhang, Z. Zhang and Y. Li, J. Mater. Chem. A, 2018, 6, 4670-4677.

33 X. Li, T. Yan, H. Bin, G. Han, L. Xue, F. Liu, Y. Yi, Z.-G. Zhang, T. P. Russell and Y. Li, J. Mater. Chem. A, 2017, 5, 22588-22597.

34 J. Yuan, Y. Zhang, L. Zhou, C. Zhang, T.-K. Lau, G. Zhang, X. Lu, H.-L. Yip, S. K. So, S. Beaupré, M. Mainville, P. A. Johnson, M. Leclerc, H. Chen, H. Peng, Y. Li and Y. Zou, Adv. Mater., 2019, 31, 1807577.

35 C. Li, H. Fu, T. Xia and Y. Sun, Adv. Energy Mater., 2019, 9, 1900999.

36 G. Wei, Z. Miao, L. Tao, M. Ruijie, A. Qiaoshi, W. Kailong, X. Dongjun, L. Zhenghui, Z. Cheng, L. Feng, Z. Fujun, Y. He and Y. Chuluo, Adv. Mater., 2018, 30, 1800052.

37 S. Liu, J. Yuan, W. Deng, M. Luo, Y. Xie, Q. Liang, Y. Zou, Z. He, H. Wu and Y. Cao, Nat. Photonics, 2020, 14, 300-305. 
38 Z. Zhou, W. Liu, G. Zhou, M. Zhang, D. Qian, J. Zhang, S. Chen, S. Xu, C. Yang, F. Gao, H. Zhu, F. Liu and X. Zhu, Adv. Mater., 2019, 32, 1906324.

39 Y. Cui, H. Yao, J. Zhang, T. Zhang, Y. Wang, L. Hong, K. Xian, B. Xu, S. Zhang, J. Peng, Z. Wei, F. Gao and J. Hou, Nat. Commun., 2019, 10, 2515.

40 L. Hong, H. Yao, Z. Wu, Y. Cui, T. Zhang, Y. Xu, R. Yu, Q. Liao, B. Gao, K. Xian, H. Y. Woo, Z. Ge and J. Hou, Adv. Mater., 2019, 31, 1903441.

41 K. Jiang, Q. Wei, J. Y. L. Lai, Z. Peng, H. K. Kim, J. Yuan, L. Ye, H. Ade, Y. Zou and H. Yan, Joule, 2019, 3, 3020-3033.

42 Y. Xu, H. Yao, L. Ma, L. Hong, J. Li, Q. Liao, Y. Zu, J. Wang, M. Gao, L. Ye and J. Hou, Angew. Chem., Int. Ed., 2020, 59, 9004-9010.

43 L. Zhan, S. Li, T.-K. Lau, Y. Cui, X. Lu, M. Shi, C.-Z. Li, H. Li, J. Hou and H. Chen, Energy Environ. Sci., 2020, 13, 635-645.

44 C. Sun, S. Qin, R. Wang, S. Chen, F. Pan, B. Qiu, Z. Shang, L. Meng, C. Zhang, M. Xiao, C. Yang and Y. Li, J. Am. Chem. Soc., 2020, 142, 1465-1474.

45 H. Lai, Q. Zhao, Z. Chen, H. Chen, P. Chao, Y. Zhu, Y. Lang, N. Zhen, D. Mo, Y. Zhang and F. He, Joule, 2020, 4, 688-700.

46 Z. Wu, C. Sun, S. Dong, X.-F. Jiang, S. Wu, H. Wu, H.-L. Yip, F. Huang and Y. Cao, J. Am. Chem. Soc., 2016, 138, 2004-2013.
47 L. Feng, J. Yuan, Z. Zhang, H. Peng, Z.-G. Zhang, S. Xu, Y. Liu, Y. Li and Y. Zou, ACS Appl. Mater. Interfaces, 2017, 9, 31985-31992.

48 J. Yuan, T. Huang, P. Cheng, Y. Zou, H. Zhang, J. L. Yang, S.-Y. Chang, Z. Zhang, W. Huang, R. Wang, D. Meng, F. Gao and Y. Yang, Nat. Commun., 2019, 10, 570.

49 X. Ma, M. Luo, W. Gao, J. Yuan, Q. An, M. Zhang, Z. Hu, J. Gao, J. Wang, Y. Zou, C. Yang and F. Zhang, J. Mater. Chem. A, 2019, 7, 7843-7851.

50 M. Luo, L. Zhou, J. Yuan, C. Zhu, F. Cai, J. Hai and Y. Zou, J. Energy Chem., 2020, 42, 169-173.

51 Y. Li, L. Zhong, B. Gautam, H.-J. Bin, J.-D. Lin, F.-P. Wu, Z. Zhang, Z.-Q. Jiang, Z.-G. Zhang, K. Gundogdu, Y. Li and L.-S. Liao, Energy Environ. Sci., 2017, 10, 1610-1620.

52 W. Li, K. H. Hendriks, A. Furlan, M. M. Wienk and R. A. J. Janssen, J. Am. Chem. Soc., 2015, 137, 2231-2234.

53 C. Zhu, J. Yuan, F. Cai, L. Meng, H. Zhang, H. Chen, J. Li, B. Qiu, H. Peng, S. Chen, Y. Hu, C. Yang, F. Gao, Y. Zou and Y. Li, Energy Environ. Sci., 2020, 13, 2459-2466.

54 A. K. K. Kyaw, D. H. Wang, V. Gupta, W. L. Leong, L. Ke, G. C. Bazan and A. J. Heeger, ACS Nano, 2013, 7, 4569-4577.

55 S. R. Cowan, A. Roy and A. J. Heeger, Phys. Rev. B: Condens. Matter Mater. Phys., 2010, 82, 245207.

56 W. L. Leong, S. R. Cowan and A. J. Heeger, Adv. Energy Mater., 2011, 1, 517-522. 